\title{
Germanica
}

\section{Philosophie als "Plagiatprofil". Wissen und Erkenntnisgrenzen der Literatur im Werk Max Frischs}

La philosophie comme "profil de plagiat ». Savoir et limites de la littérature dans l'œuvre de Max Frisch.

Philosophy as a "pattern for plagiarism". Knowledge and its Limits in Literature in Max Frisch's Works.

\section{Walter Schmitz}

\section{OpenEdition}

Journals

Édition électronique

URL : http://journals.openedition.org/germanica/1182

DOI : $10.4000 /$ germanica. 1182

ISSN : 2107-0784

\section{Éditeur}

Université de Lille

Édition imprimée

Date de publication : 1 juillet 2011

Pagination : 55-74

ISBN : 9782913857278

ISSN : 0984-2632

\section{Référence électronique}

Walter Schmitz, "Philosophie als "Plagiatprofil". Wissen und Erkenntnisgrenzen der Literatur im Werk Max Frischs », Germanica [En ligne], 48 | 2011, document 4, mis en ligne le 01 juin 2013, consulté le 06 octobre 2020. URL : http://journals.openedition.org/germanica/1182 ; DOI : https://doi.org/10.4000/ germanica. 1182

Ce document a été généré automatiquement le 6 octobre 2020.

(C) Tous droits réservés 


\title{
Philosophie als "Plagiatprofil". Wissen und Erkenntnisgrenzen der Literatur im Werk Max Frischs
}

\author{
La philosophie comme " profil de plagiat ». Savoir et limites de la littérature \\ dans l'œuvre de Max Frisch. \\ Philosophy as a "pattern for plagiarism". Knowledge and its Limits in Literature \\ in Max Frisch's Works.
}

\section{Walter Schmitz}

1 Nachdem sich die beiden ursprünglich als Schweizer Dioskuren gefeierten Autoren längst gründlich "auseinander befreundet" hatten ${ }^{1}$, hat Friedrich Dürrenmatt noch immer eine Eigenart des Schriftstellers Max Frisch genau erkannt. In einem - freilich boshaft karikierenden - Interview hat er Frisch 1990 als den "Schriftsteller der Intellektuellen" bezeichnet, Intellektualität aber vor allem auf "Ehe- und Identitätskrisen“ verkürzt ${ }^{2}$. Was in Frischs Werken das Motivinventar des, wirklichen Lebens ${ }^{\star}$ - also Ich-Erfahrung und Erkenntnis im Kontinuitätszusammenhang einer unverwechselbaren, in Liebe kommunizierbaren Lebensgeschichte - darstellt, das wird, in Dürrenmatts Sicht, auf private Verwicklungen und Identitätsnöte des Autors reduziert. Die grundsätzlichen Vorwürfe hat der Kontrahent gelegentlich variiert, Frischs ,Narzißmus' und - daraus folgend - "Unwahrheiten" monierend ${ }^{3}$. Sich selbst hingegen präsentierte Dürrenmatt als einen Schriftsteller, der sich für den gesamten literarischen Betrieb überhaupt nicht interessiere und allenfalls "naturwissenschaftliches oder philosophisches" lese ${ }^{4}$. Jene Passagen über Max Frisch, denen sich ähnliche über andere Autoren wie Günter Grass oder Heinrich Böll im selben Interview beigesellten, lösten einen solchen Skandal aus, daß der mit Dürrenmatt befreundete Heinz Ludwig Arnold in einem zweiten Interview um Klärung bemüht war, und jetzt setzt Dürrenmatt als Schlußpunkt eben die Differenz seiner eigenen Position gegenüber all den anderen von ihm kritisierten : Er sei jemand, der über Dinge nachdenke, "über die die heutigen Schriftsteller nicht nachdenken. Du weißt, daß ich mich hauptsächlich für Philosophie, für Naturwissenschaften und sehr 


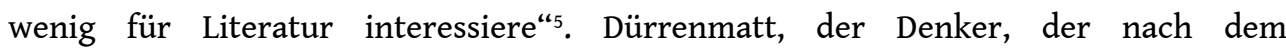
Zusammenhang der Welt fragt - Frisch, der Literat, der sich in seinen Privatheiten verstrickt : Dieses Doppelbildnis liegt dem Auftritt des Zeugen Neuburger (Dürrenmatt lebte in Neuburg an der Donau) in der Erzählung Blaubart voraus. Die Rolle des Zeugen im Frisch-Werk ist ja - wie bereits im Bühnenstück Andorra die selbstgerechten Auftritte der mitschuldigen Andorraner, die an der Zeugenschranke ihre Unschuld beteuern, längst belegten - ziemlich zweifelhaft. Neuburger folgt schwadronierend Dürrenmatts Interviewäußerungen, jetzt bezogen auf den ,PseudoBlaubart' Felix Schad, der zu Unrecht des Mordes an seiner Ehefrau angeklagt ist - auch eine Art Ehekrise -, und gipfelt in der Bemerkung: "Er kann halt nicht denken." ${ }^{6}$ Ein ,Philosoph' also ist, diesem Zeugen zufolge, der Angeklagte Felix Schad ebenso wenig wie Max Frisch in der Einschätzung Dürrenmatts zu eigenständigem Denken, wie es die Philosophie verlangt, fähig wäre.

\section{Philosophie im "Zeitalter der Reproduktion"}

2 Freilich steht, wenn es um ,Max Frisch und die Philosophie' geht, durchaus Mannigfaches zur Verhandlung. Vorab seien deshalb die Dimensionen benannt, in die sich der Sammelbegriff ,Philosophie' in einer Lektüre von Max Frischs Werk auffächert ; und zwar läßt - zunächst - Philosophie sich als eine institutionell verfaßte Form menschlichen Wissens verstehen, mit - des weiteren - einer langen Tradition von Einsichten, Modellen, Systemen, die einzelnen Philosophen zuzurechnen sind. Das Wissen, die Philosophie, die Philosophen - danach ist also jeweils besonders zu fragen, wenn man Max Frischs Verhältnis zur Philosophie genauer begreifen will.

3 Walter Faber, der Protagonist des vielleicht erfolgreichsten Romans von Max Frisch, Homo Faber, insistiert auf seiner Identität und weiß, wo die ,Wahrheit' zu finden ist : "Ich erklärte, was die heutige Kybernetik als Information bezeichnet" und was "die Höchstgeschwindigkeitsrechenmaschine“ an Zukunftserkenntnis zu generieren vermag, um die Zeit des Lebens kalkulierbar zu machen : "Der Roboter erkennt genauer als der Mensch, er weiß mehr von der Zukunft als wir, er errechnet sie." ${ }^{\text {“7 }}$ Fü Faber ist Philosophie Metaphysik und ,Mystik', und die Abwertungsformeln gegen alles, was sich menschlicher Praxis entzieht, sind reihenbildend in seinem autobiographischen ,Bericht', der ja zugleich der Romantext ist. Allerdings wechselt der Referenzrahmen : "Ich brauche [...] keinerlei Mystik; Mathematik genügt mir", heißt es etwa an anderer Stelle ${ }^{8}$, und mit Mathematik ist hier jedenfalls die anwendungsfähige Mathematik gemeint, welche jenen technischen Leistungen, welche dem Laien wie ein Wunder vorkommen mögen, die rationale Grundlage bietet.

4 Auch Stiller, der Protagonist im vorhergehenden Roman Max Frischs, zu dem der nachfolgende Homo Faber in genauer Komplementarität entworfen wurde, vermag der Philosophie wenig abzugewinnen. Als Künstler sind ihm - anders als dem Techniker Faber - die Berühmtheiten der Geisteswissenschaften zwar auch namentlich vertraut, und dem Appell ihrer Sinnangebote vermag er sich nicht so lakonisch zu entziehen, wie dies seine technisch orientierte Komplementärfigur unternimmt. Daher fällt Stillers Abwehr umfassender und prägnanter aus als die abfälligen Formeln Walter Fabers; sie kann freilich die Verzweiflung des Ich-Erzählers, jenes Mr. White, der vorgibt, nicht Stiller zu sein, aber zuletzt mit diesem identifiziert wird, nicht verhehlen. Als ,White' beruft sich der Erzähler auf eine Authentizität der Selbsterfahrung, die ihm selbst 
zugleich zweifelhaft sein muß, denn - wie er sich eingesteht: "Wir leben in einem Zeitalter der Reproduktion"; die Außenwelt müsse man gar nicht, wie es ,White' doch unternommen hatte, selbst erfahren, ihre mediale Vermittlung überforme "unser[] persönliche[s] Weltbild" ohnehin; dies gelte ebenso für das "menschliche[] Innenleben“, zu dem ein authentischer Zugang durch massierte Deutungsklischees produziert von einer Tiefenpsychologie à la C.G. Jung oder auch einer katholischen Existenzanalyse à la Bernanos - versperrt sei ; und dies betrifft auch die Philosophie. Da jeder "in lauter Plagiaten" lebe und erlebe, sei es "in diesem Zeitalter schon eine Rarität, einen Kopf zu treffen, der auf ein bestimmtes Plagiatprofil gebracht werden kann, es zeugt von Persönlichkeit, wenn einer die Welt etwa mit Heidegger sieht und nur mit Heidegger ${ }^{\star 9}$. Gleichwohl wird Stiller der Philosophie nicht entnommen.

\section{Philosophie und Lebens-Sinn}

5 Kritisch und derart distanziert war allerdings das Verhältnis des Autors Max Frisch zur Philosophie keineswegs immer gewesen. Daß der Germanistik-Student aus kleinbürgerlichem Hause, der zudem in der kulturkonservativen Neuen Zürcher Zeitung als Feuilletonist ein Unterkommen suchte, daß sich also der junge Max Frisch höchst affirmativ zu allen in seiner kulturkonservativen Vaterstadt hoch geschätzten Kulturgütern verhielt, stand zu erwarten. Die kulturelle Überlieferung im Wort, also vor allem Literatur und Philosophie, bedeutet für ihn selbstverständlich Maß und Wert. Debatten, die er noch in den 1940er Jahren mit seinem Freund Werner Coninx führt, verweisen auf die traditionell bildungsbürgerliche Konstruktion eines unpolitischen Reiches der Kultur, das nicht nur autonom, sondern vor allem auch Reich des Höheren und des Ideals sei. In diesen 1940er Jahren freilich fühlt Max Frisch sich schon von der ,Forderung des Tages' ${ }^{\prime 10}$ herausgefordert. Zugleich umspannt dieses Jahrzehnt etwa jene Phase, in der Frisch die Traditionen, die er bis zu dem Autodafé seiner literarischen Versuche und bis zur Aufnahme seines Architekturstudiums unkritisch verehrend adaptiert hatte, nun im einzelnen überprüft und dabei gleichsam diejenigen Kontexte, die für sein Æuvre bestimmend sein werden sollen, zum ersten Mal markiert. Es bleibt dabei freilich bei synkretistischen Konstruktionen, und es geht dem Autor offenbar nicht darum, eine spezifische Position so entschieden zu konturieren, daß es zu einer unmittelbaren, kritischen Auseinandersetzung - von Nachfolge ganz zu schweigen mit einem maßstabsetzenden Vorbild kommen könnte. Es geht eher darum, für Gedanken und Motivfiguren Anregungen und Präzisierungen in der Tradition zu finden, und in diesen Suchbewegungen werden nun auch allmählich jene philosophischen Werke kenntlich, die Max Frischs Euvre für eine ganze Weile begleiten werden ${ }^{11}$.

6 Es ist zu Beginn noch Nietzsche, dessen Denken den Schriftsteller Max Frisch herausfordert - so im Roman Die Schwierigen oder J'adore ce qui me brûle.Freilich sind Nietzsches Konzepte bereits in diesem zweiten Roman Frischs kompatibel gemacht mit Ansätzen anderer: "Nietzsches Kastengliederung des Humanen, Schopenhauers pessimistische Philosophie und Weiningers Geschlechtsangst, die den Selbstmord anrät, erledigen“, so habe ich in einer früheren Studie formuliert, "das Problem des ,halben Menschen' "12 - und zwar des schwierigen Protagonisten Jürg Reinhart. Nicht der Erzähler, sondern Reinhart formuliert die Einsicht, die er für wegweisend hält : 
Eigentlich gibt es nur drei Wege für jeden Menschen [...]. Er darf das Erbe seiner Herkunft, das Ergebnis von Geschlechtern, [...] in der Vergeudung einer einzigen Rakete verbrennen, rücksichtslos, wenn er hoch genug reicht! Das sind die Gestalter des Lebens, jene, die eine Fessel nicht nötig haben, sich selber den Sinn geben - im Übermut [...] zählte ich mich einmal selber dazu ! [...] Das andere sind die Gesunden, die Erhalter des Lebens, das sie weiterbieten, wie sie es empfangen haben, so unversehrt als möglich, das ist die bürgerliche Ehe. [...] Das Dritte, [...] man hat sein Leben so versehrt empfangen, daß man sich selber damit auszulöschen hat. ${ }^{13}$

7 Zugleich freilich distanziert sich der Autor von diesen Meinungen seines Protagonisten durch deutliche Ironiesignale. Reinhart meint - offenbar von anderen Passagen aus Nietzsches Schriften inspiriert ${ }^{14}-$, durch seine Selbstauslöschung zugleich doch noch Anteil an ,adligem Menschentum' zu erreichen, und ist sich darin mit seinem Gegenpart, einem Schweizer Oberst, einig. Doch Reinharts Versuch, sein Sterben in den großen Sinnzusammenhang der Natur einzubauen, in dem alles wiederkehre, mißlingt. Denn präzise ist der Ort des Menschen in der Natur erst im Paradox gefaßt, und diese paradoxe Struktur wird in dem Leitsatz von J'adore ce qui me brûle abgebildet : "Nichts kehrt uns wieder, alles wiederholt sich. ${ }^{\text {“15 }}$ Denn die große Wiederholung gilt wohl der Ordnung der Natur; dem Menschen aber bleibt nur die Einmaligkeit seines Todes. Damit ist zugleich jenes Dilemma benannt, das die produktive Nietzsche-Rezeption Max Frischs beenden wird. Nietzsches Philosophie von der ewigen Wiederkehr des Gleichen hatte versucht, "[...] ein finalistisches Sinnkonzept und das Kausalprinzip der Naturwissenschaften zu verschmelzen“, also gleichsam das Paradox im Leitmotiv der Schwierigen in einer innerweltlichen Transzendenz aufzuheben: "Der ,Wille zur Macht' sollte mehr sein als blanker Naturtrieb, das Individuum sollte mehr sein als ein ,naturalisiertes Wesen', das sterben muss. Die Lehre von der ewigen Wiederkehr des Gleichen sollte das anthropologische Paradox, das Endlichkeit und Unendlichkeit zugleich umfaßt, innerweltlich erhalten - nach der Verkündigung vom Tode Gottes und nach der Demontage der christlich-,hinterweltlerischen' Transzendenz. ${ }^{16}$ Max Frisch wird diese immanente Transzendenz, wie sie von Nietzsche entworfen wurde, künftig nicht mehr gelten lassen, und wenn nach Kriegsende noch einmal Thesen Nietzsches in Frischs Werk pointiert vertreten werden, so handelt es sich um das verbrecherische Gedankenexperiment des Nietzscheaners und SS-Manns Herbert, der meint, durch Frevel Gott herausfordern zu können ${ }^{17}$. Die genuine Philosophie Nietzsches steht hier freilich gar nicht mehr zur Debatte. Herbert, der Nihilist und ,bleiche Verbrecher', den Nietzsche selbst schon als Negativfigur charakterisiert hatte, findet sein Komplement im Oberlehrer, der Personifikation des Nietzscheschen ,Bildungsphilisters'. Von Philosophie ist hier nicht die Rede, sondern von ihrer Vernutzung und Entwertung.

Gegen Ende der Nachkriegszeit ist dann der Beitrag Nietzsches gleichsam aufgehoben in den Resultaten, die sich aus Frischs Auseinandersetzungen mit dem Existentialismus, insbesondere Jean-Paul Sartres, ergeben. Die Perspektivik der Weltwahrnehmung, d.h. das Verfertigen von Bildern und die notwendige Partikularität solcher ,Bildnisse', das sind Prämissen von Überlegungen Frischs, die in der Rezeption zu einer ,Bildnistheorie' avancierten : "Du sollst dir kein Bildnis machen, heißt es, von Gott. Es dürfte auch in diesem Sinne gelten: Gott als das Lebendige in jedem Menschen, das, was nicht erfaßbar ist. Es ist eine Versündigung, die wir, so wie sie an uns begangen wird, fast ohne Unterlaß wieder begehen - / Ausgenommen wenn wir lieben ${ }^{18}$." Sich jetzt noch streng auf Nietzsche zu berufen, war für den Autor des Tagebuchs 1946-1949 schon deshalb überflüssig, weil es für ihn längst um ein - synkretistisches - Konzentrat aus 
vielfältigen Anregungen ging ${ }^{19}$ - wie manchem, das schon in der Nietzsche-Rezeption des Vitalismus zirkulierte ${ }^{20}$, dann aber auch Bertolt Brechts Überlegungen Über das Anfertigen von Bildnissen ${ }^{21}$ und vor allem dem nach 1945 allerorten diskutierten Existentialismus eines Albert Camus und eines Jean-Paul Sartre ${ }^{22}$.

So läßt sich jenes ,Bildnisverbot', wie es zuerst im Tagebuch 1946-1949 formuliert wurde, "diese Negativform einer Lehre", durchaus auffassen als die "moralistischethische Weiterentwicklung jener Aussagen Sartres über die Rolle des anderen als Bestandteil der Situation, die sich im Hauptwerk Das Sein und das Nichts (1943) sowie den Frisch bekannten Betrachtungen zur Judenfrage (1945) finden ${ }^{23}$." Die Skizze Der Andorranische Jude hatte im Tagebuch ja als Exempelerzählung gedient, aus der sich die Maximen zum Bildnis ableiten ließen ${ }^{24}$. Max Frisch aber entwickelte damit in den 1940er Jahren eine Perspektive auf die ,Philosophie', die für sein gesamtes weiteres Schaffen bestimmend bleiben wird, dabei gleichsam die Kritik der Systemphilosophie umsetzend, die im 19. Jahrhundert begonnen hatte; nicht das umfassende System und die allgemeine Systematik, sondern der Mensch und seine jeweilige Erfahrung rücken ins Zentrum dieses Philosophierens ${ }^{25}$ - eines Kierkegaard, eines Schopenhauer, eines Nietzsche und weiter über die deutsche Existenzphilosophie - hier muß auch der Name Heideggers genannt werden - bis zum französischen Existentialismus. Und angesichts der Prominenz, die Sartre und auch Camus in der Nachkriegszeit und noch weit in die 1950er Jahre hinein zugewachsen war, darf man den französischen Existentialismus wohl mit Fug und Recht "als Angelpunkt für Frischs Weltentwurf" ${ }^{26}$ bezeichnen. Eine präzise Rezeption findet gleichwohl nicht statt, sondern es werden gleichsam umstrittene Konzepte übernommen ${ }^{27}$ und in das literarische Experiment Max Frischs eingeführt, so etwa das humane Kriterium der Wahl, die Rolle des, anderen' in einer Situation, die Selbstbelügung im ,mauvaise foi', die man Stiller vorwerfen könnte, und die Welthypostase im "esprit de sérieux", die Fabers komplementäre Verfehlung wäre ${ }^{28}$.

\section{Parodierte Existenzphilosophie / Gescheiterte Existenz}

Für die Figuren in Frischs Werken der 1950 Jahre, die in die Bahnen der Existenzphilosophie einschwenken, bedeutet dies den direkten Weg zum Scheitern ihrer Existenz. Dies gilt in beiden Varianten : sei es, daß sie ihre eigene, je besondere Situation mit Termini analysieren, sie mit Hilfe von Konzepten zu verstehen suchen, die philosophische Vorfahren haben und für die philosophische Gewährsleute angeführt werden könnten oder auch angeführt werden. Oder sei es, daß sie in ihrer vorgeblich höchstpersönlichen Existenz gleichsam die Konzepte der Existenzphilosophie zitieren und, ohne es zu wissen, ihnen nachleben - im ,Plagiatprofil'. Es scheint geradezu, daß in dieser Werkgruppe der 1950er Jahre Max Frisch eine Art parodistische Intarsienarbeit betreibt, um die Denkfiguren und Wissenselemente nicht nur in den Weltentwurf, sondern begründend in die Geschichte des Scheiterns der Protagonisten mit hineinzunehmen.

11 Beide Varianten verschränken sich miteinander. Als man Mr. White, den Schreiber der Tagebuchhefte, aus denen Frischs Roman Stiller besteht, immer härter bedrängt, wieder Stiller zu werden, sich sein Leben als Stiller also wiederzuholen und es damit auch zu wiederholen, notiert er zunächst scheinbar ganz einsichtig ${ }^{29}$ : 
Wiederholung! Dabei weiß ich : alles hängt davon ab, ob es gelingt, sein Leben nicht außerhalb der Wiederholung zu erwarten, sondern die Wiederholung, die ausweglose, aus freiem Willen (trotz Zwang) zu seinem Leben $\mathrm{zu}$ machen, indem man anerkennt: Das bin ich !... Doch immer wieder (auch darin die Wiederholung) genügt ein Wort, eine Miene, die mich erschreckt, eine Landschaft, die mich erinnert, und alles in mir ist Flucht, Flucht ohne Hoffnung, irgendwohin zu kommen, lediglich aus Angst vor Wiederholung -

Daß Wiederholung ein Schlüsselkonzept der Existenzphilosophie seit Kierkegaard ist, muß bei Stillers Angst nicht mitgedacht werden. Kierkegaard hatte jedoch der ,Wiederholung', die er auf theologischer Grundlage konzipiert, eine eigene Schrift mit diesem Titel gewidmet ${ }^{30}$. Kierkegaards Konzept wird aber in Stillers markantem Stichwort nur aufgerufen, jedoch dann nicht eingelöst. Ähnliches gilt für Heideggers "Analyse der Zeitlichkeit des menschlichen Daseins" ${ }^{\text {"31 }}$. Stiller weigert sich ja geradezu, die eigene Geschichte wieder vor-sich-zu-holen, also in diesem Heideggerschen Sinne $\mathrm{zu}$,wiederholen'. Im jüngeren französischen Existentialismus, bei Sartre zumal, wird ,Wiederholung' nicht konzeptionell ausdifferenziert, muß jedoch im Spannungsfeld von Freiheit und Determination des Subjekts mit gedacht werden, und dies gilt erst recht für die von Camus entworfene mythische Figur des Sisyphus, die eben in der Wiederholung als Zeitfigur des Absurden zur Selbstannahme findet. So läßt sich diese Passage im Stiller, der weitere anzufügen wären, zwar wie ein Palimpsest in verschiedene Schichten existenz-philosophischer Reminiszenzen auflösen, weitere Präzisierung ist aber wegen dieser Palimpseststruktur nicht möglich, wäre wohl auch im semantischen Raum des Romans als ,Plagiatprofil' zu qualifizieren. Ähnlich ließe sich in Stillers Agieren als White eine Entsprechung zu dem finden, was Jean Paul Sartres Essay Ist der Existentialismus ein Humanismus? als Bedingung authentischer Existenz formuliert hatte - "daß der Mensch ohne irgendeine Stütze und ohne irgendeine Hilfe in jedem Augenblick verurteilt ist, den Menschen zu erfinden “"32; Stiller freilich erfindet sich nur selbst.

13 Solche "Selbstsetzung" als existentielle Geste kennzeichnet auch andere Figuren Frischs, den Staatsanwalt, der sich in ,Graf Öderland' verwandelt, und noch jenen Herrn Kürmann, der frei - oder doch : willkürlich - sein Leben wählen will. Stets erfinden sie allerdings auch die Lebensgeschichten anderer und fallen damit unter das Verdikt des Bildnismachens, so wie bereits Stiller es sich zum Vorwurf machen lassen mußte. Wenn ihn freilich die schöne Julika, seine Ehefrau, damit konfrontiert, so schrumpft das biblische Pathos des Verbotes zum Pseudo-Argument in einem läppischen Ehestreit :

,So also siehst Du mich !' sagte Julika. ,Du hast dir nun einmal ein Bildnis von mir gemacht, das merke ich schon, ein fertiges und endgültiges Bildnis, und damit Schluß.' [...] ,- nicht umsonst heißt es in den Geboten : du sollst dir kein Bildnis machen! Jedes Bildnis ist eine Sünde. Es ist genau das Gegenteil von Liebe, siehst $\mathrm{du}$, was du jetzt machst, mit solchen Reden. Ich weiß nicht, ob du's verstehst.' [...] ,Woher hast Du das ?' fragt er nur. ${ }^{33}$

Die Quelle für "diesen nicht unbekannten Gedanken" darf man in jenem bibelkundigen kleinen ,Jesuiten' vermuten, mit dem Julika auf ihrem persönlichen ,Zauberberg'

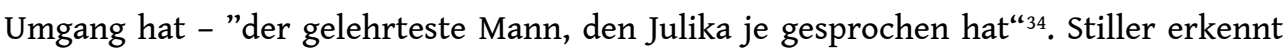
denn auch, daß hier von leidvoller existentieller Erfahrung weniger die Rede sein kann denn von Lektürefrüchten. Auch dem Staatsanwalt Rolf in seinem "Nachwort" ist die ,Bildnistheorie' geläufig, und da er inzwischen selbst aus den leidigen Verstrickungen von Stillers Geschichte salviert und wieder, wie es sich gehört, glücklicher Gatte, angesehener Bürger und gebildeter und vernünftiger Mensch ist, weiß er sie souverän 
auf den problematischen Stiller anzuwenden ${ }^{35}$. Die Eheleute aber, Stiller wie Julika, wiederholen nur noch im Zitat ein ihnen längst schon abhanden gekommenes authentisches Leben. Authentisch ist nur ihre Selbstverfehlung - wie die daraus zwanghaft resultierende Verfehlung des anderen. $\mathrm{Zu}$ der erfüllten Lebensfigur Kierkegaards - jener Wiederholung, die der Prüfstein der Freiheit ist ${ }^{36}$ - verhält sich die Wiederholungsfigur, in die Whites/Stillers Leben zuletzt doch einmündet, somit bloß noch wie eine mechanisch tödliche Repetition ${ }^{37}$. -Soweit allerdings ist die Präsenz Kierkegaards in Stillers Leben nicht ausgeschöpft, wird ihm doch der Deutungsanspruch des Existenzphilosophen noch einmal von berufener Seite nahe gebracht. Im "Nachwort" des Staatsanwaltes, der sich selbst jetzt in der Rolle des schreibenden Freundes sieht ${ }^{38}$, erfahren wir, er habe dem in schwierigen Verhältnissen wieder mit Julika zusammen Lebenden auf dessen Wunsch hin "eine umfängliche Büchersendung" geschickt, darunter ein Band Kierkegaard, freilich "so schweres Zeug" ${ }^{39}$, daß Stiller ihn, wie er gelegentlich erwähnt, nicht immerzu "lesen konnte ${ }^{\text {“40 }}$. Rolf jedoch dekliniert die Stadien der Selbstannahme gleichsam in Anwendung Kierkegaardscher Scholastik auf Stillers Lebensweg, "von der Melancholie der bloßen Selbsterkenntnis" zur "Resignation" bis zur "Selbstannahme“" ${ }^{\text {" }}$. Und schließlich steht noch der Sprung in den Glauben zu erwarten, "die Gewißheit, daß unser Leben von einer übermenschlichen Instanz gerichtet wird, und zugleich wenigstens die leidenschaftliche Hoffnung, daß es diese Instanz gäbe ${ }^{42}$." Gerade jenes existentielle Pathos, das bei Kierkegaard den Sprung in den Glauben einleitet, verlangt Rolf in einer letztlich resultatslosen nächtlichen Aussprache angesichts der "schlappe[n] Resignation" ${ }^{43}$, die er bei Stiller diagnostiziert : "Zittere! Du weißt schon, wie ich das meine“" ${ }^{\prime 4}$ - auch hier also wieder die scheinbar selbstverständliche Verweisfloskel auf Bekanntes, nämlich eben jenen übersandten "Band Kierkegaard“.

15 Niemand hingegen predigt Walter Faber, dem Mann der Technik, die philosophische Wahrheit seiner Existenz. Die subversive Schreibweise des Romans Homo Faber allerdings, so wie sie schon vielfach nachgezeichnet wurde, bezieht aus der Philosophieverweigerung des Protagonisten ihren Rückhalt und stellt sie zur Disposition. Denn tatsächlich begegnet Faber dem Lebensanspruch des ,Geistes', wie er in der Philosophie seine institutionalisierte Form findet, allenthalben - auch wenn in seinem Weltbild der ,Geist' nur als "Maxwellscher Dämon" seinen Platz hat. Daß er so selbstgewiß die ,Mathematik' gegen die ,Mystik' zu setzen vermag, wäre - auf der Textebene - niemals ohne jenen Großroman Robert Musils Der Mann ohne Eigenschaften möglich, den man mit Fug und Recht als einen philosophischen Roman bezeichnen darf. ${ }^{45} \mathrm{Ganz}$ im Sinn des Ganzheitsbegehrens der Kultur um 1900 - das seinen Rückhalt nicht so sehr in der Schulphilosophie denn in Denkfiguren einer ,Weltanschauung' findet - hatte Musil ja die Formel ,Mathematik und Mystik' geprägt und damit den defizienten Status des Weltbildes eines Homo Faber vorab definiert. Mehr noch aber : Sabeth, das junge Mädchen, dem er auf seiner Schiffsreise über das Meer des Lebens begegnet, wird von ihm selbst als personifizierte Agentin des Philosophischen wahrgenommen - mit ihrem "schwarzen Pullover mit Rollkragen,

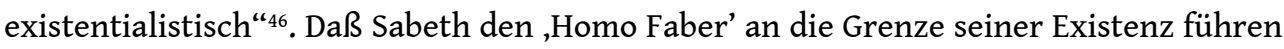
wird, ist nur folgerichtig.

Der Roman entwickelt aus diesem Ansatz des Existentialismus zudem eine mythische Tiefenstruktur, in einer Textbewegung, die jene Geste der Reinterpretation des Archaischen als Existential, wie sie Sartre in seinen in Deutschland in den 1950er 
Jahren nachgerade modisch gewordenen Dramen vorgenommen hatte ${ }^{47}$, genau nachzuvollziehen scheint. Die Kritik hat diesen Sieg des existentiellen Mythos über die Technikerrolle im Homo Faber denn auch geradezu enthusiastisch gefeiert. Allerdings bleibt zu bemerken, daß die existentialistische Philosophie im Roman zunächst einmal in der Form des modischen Accessoires auftritt, Sabeth eher als Ikone existentialistischer "Populärphilosophie“ erscheint ${ }^{48}$. Von einer genuinen Auseinandersetzung mit Sartres Denken oder auch mit dem von Albert Camus kann nicht die Rede sein.

17 ,Philosophie' wird so in der Literatur Max Frischs aufgelöst in ein Inventar von Sinnangeboten, die vor der jeweiligen Erfahrung, wie sie Frischs Erzählexperimente des Lebens herausarbeiten, zuschanden werden: Philosophische Konzepte vermögen die jeweilige Erfahrung zum Tode, welche die Protagonisten zu bestehen hätten, nicht hinreichend zu erklären, und grade wenn sie mit Schlüsselworten und Kennzitaten im Text angeboten werden, versagen sie als Deutungsangebote erst recht. Wenn gar die Figuren selbst im Debakel ihrer Existenz Rat bei der Philosophie suchen, geraten sie vollends in schablonenhafte und damit nicht mehr angemessene Konventionen des Handelns und Denkens. Sie verstricken sich - laut Frischs Eigenterminologie - im ,Bildnis' noch dann, wenn sie das ,Bildnis' zu erkennen meinen.

Bereits vor den beiden großen Romanen hatte Frisch in einer Version des Don-JuanStoffes, dem Drama Don Juan oder die Liebe zur Geometrie, jene beiden Daseinsentwürfe, für die die Philosophie ihm Denkentwürfe anbot, enggeführt : auf der einen Seite das Verlangen nach Erkenntnis, für das wiederum die Chiffre der Mathematik einsteht, nun freilich nicht im Sinne des Homo Faber, sondern im Sinne des Spinoza und Descartes, ein Leben gleichsam more geometrico, gemäß einem ,Wissen, das stimmt ${ }^{\prime 49}$. Das wäre - in Frischs Lesart - das Ideal jenes jungen Mannes namens Don Juan gewesen, der dann ironischerweise als Personifikation des großen Verführers in die Geschichte abendländischer Kultur einging, der also die Wahrheit seiner Existenz in der Dämonie autonomer Sinnlichkeit verfehlte ; so hatte Kierkegaard es an der Figur des Verführers gezeigt ${ }^{50}$. Und in der Tat ist der Quellenbezug zu Sören Kierkegaards Schriften in Don Juan oder die Liebe zur Geometrie so eng wie selten zwischen einem Werk Max Frischs und einem philosophischen Referenztext. Freilich richtet sich dieser Bezug eben nicht auf die genuine Philosophie Kierkegaards, sondern auf eine Warnfigur, die all das verkörpert, was jener Philosophie zuwider läuft. In Frischs Drama wird Kierkegaards Polarität von Sinnlichkeit und Transzendenz anthropologisch umgedeutet zu einer jedenfalls immanenten Polarität von Geist und Körper. Frischs Don Juan muß feststellen, daß seine erotische Lebensverfehlung nur durch mythisierende Inszenatorik überhaupt zu korrigieren, wenn auch nicht zum ,Geist' hin zu retten ist. Nur indem er der Legende seinen Tribut zollt und seine eigene ,Geister'-Erscheinung und Höllenfahrt inszeniert, vermag er überhaupt den Erwartungen ,der Damen', also dem gesellschaftlich produzierten „Bildnis', zu entkommen, aber eben nicht zum reinen Geist, sondern zur Rolle eines Liebhabers der souveränen Hure Miranda, die es mittlerweile zur Herzogin von Ronda gebracht hat und dem Gescheiterten eine Zuflucht bietet, damit zugleich jene Gegenwelt des Körpers, gegen die Don Juan so energisch angegangen war, endgültig als die eigentliche etablierend. Sogar die Kirche ist mit im Komplott. 


\section{Erfahrung, Wissen und die Philosophen} ,prekären Konstellation zum Leben'. Daß die Literatur das Leben nicht zu erreichen vermöge, resultiert für ihn aus den Schreibexperimenten der 1940er Jahre : "Man sagt, was nicht das Leben ist, man sagt es um des Lebens willen ${ }^{51}$ " Letztlich, und dies ist die poetologische Übersteigerung des ,Bildnisverbotes', wird alles sprachlich Formulierte zum Bildnis, letztlich sogar jedes Wort, und für die Gehalte der Philosophie gilt dies ohnehin. - Andererseits läßt auch gerade die notorische Erkenntnisskepsis in Frischs Werken, zu der sich auch deren Autor beharrlich bekannt hat, als die Kehrseite einer Faszination von wahrer Erkenntnis entziffern.

Der Erkenntniszweifel Frischs richtet sich allerdings auf die Philosophien in doppelter Weise - einmal eben auf ihre sprachlich fixierte Form, zum anderen aber auf ihre Geste der Objektivierung, die selbst dann, wenn die Existenz des Menschen und das Subjekt im Zentrum stehen, aus der institutionellen Verfaßtheit des philosophischen Denkens resultiert. Die authentische Erfahrung liegt jeder Philosophie voraus.

Frage nach dem Wissen aber formuliert Max Frisch seit seinen Anfängen mit der Kennvokabel des Geistes, die er aus der vitalistischen Tradition um 1900 entnommen hat. Die gängige Formel ,Geist versus Leben' wurde kulturkritisch zu ,der Geist als Widersacher des Lebens' verschärft ${ }^{52}$ und wird auch so vom jungen Max Frisch übernommen. Der Geist, das ist der Anspruch des Absoluten. Daß die Begegnung mit dem ,Geist' das ,Leben' völlig umwandeln müsse, ist das Thema der Parabelerzählung $S_{S c h i n z}{ }^{53}$, in der ein Rechtsanwalt (also der Vertreter eines ideal-normativen Anspruchs, der aber gesellschaftlich institutionalisiert und damit auch fälscht) sein gesamtes Leben ändert und, wie die Bürger spöttisch-abschätzig bemerken, ein Linksanwalt wird. Also einer, der für die wahre Gerechtigkeit in einer Gesellschaft eintritt, die so viel Wahrheit nicht erträgt.

Max Frisch hatte übrigens, als das Verhältnis zu Dürrenmatt noch freundschaftlich verstehend war, eben diesen Anspruch im Schaffen des anderen erkannt und ihn als Experiment des - christlichen - Glaubens identifiziert, denn der letzte Rückhalt des Wissens ist die Religion. Diese Prämisse ist für Frisch allerdings ebenso unausweichlich wie unerfüllbar. Christliche Motivik und Thematik wird in seinen Texten genutzt, um diese unauflösbare Paradoxie anzuvisieren. So verläuft denn im Stiller neben den recht offenkundigen Anspielungen auf philosophiegeschichtlich fixierte Positionen ein Subtext, der auf jene leere Stelle in diesem Romanaufbau zuläuft: das Scheitern geistiger Erkenntnis, parodistisch gespiegelt. Es geht um das ,Rätsel' zwischen Stiller und der "schönen Julika" ${ }^{\text {"54 }}$, das unlösbar ist, da eine Erlösung nicht eintreten kann, nicht einmal an Ostern, dem christlichen Erlösungsfest : Es ist Julikas Todestag ${ }^{55}$. - Die weitere Deutung, vom Thema und Leitwort ,Erlösung' ausgehend, hätte vor allem den Doppelsinn von Erkenntnis, der dem Wort bereits in der deutschen Bibelübersetzung eignet, als sexuelle und intellektuelle Vereinigung zugleich hier zu entfalten, sie würde damit jene Dimension erschließen, welche die Motivkonfiguration des Geistes - und damit auch der Philosophie - in Frischs Werk stets konterkariert: diejenige der Liebe als Utopie und diejenige der Sexualität als Zentrum männlichen Versagens.

Existentielles Wissen wird dennoch nicht von der Philosophie verwaltet, bildet aber ihre einzige Legitimation - und den Maßstab ihrer Problematisierung durch Literatur. In Frischs Spätwerk ist diese Koppelung dann weitgehend gelöst. Die existentiellen 
Kennworte wie ,Wahl' und ,Wiederholung' strukturieren weiter die Texte, doch der Bezug auf die Existenzphilosophie verblaßt ${ }^{56}$. Wohl findet sich in Mein Name sei Gantenbein noch eine Anspielung auf Sartres Les Jeux sont faits : "Das Spiel ist aus", lautet eine formelhafte Einfügung mit unmittelbarem Titelverweis ${ }^{57}$. Aber die Distanznahme macht selbst vor jenen zentralen Konzepten nicht Halt, die nun gleichsam in Frischs Werk selbst umstritten werden. Hieß es im Tagebuch noch : "Die Würde des Menschen [...] besteht in der Wahl “"58, ein Satz voller Reminiszenzen an den französischen Existentialismus, so ist Herr Kürmann, dessen Name ja bedeutet, er sei einer, der wählen könne, jetzt, 1967, im Bühnenstück Biographie. Ein Spiel zwar zu vielfältigen Wahlakten in der Lage, die freilich an der Struktur seiner Erfahrung scheitern. Denn er kommt immer wieder auf dieselbe Geschichte zurück $^{59}$; daß aber die Frau, Antoinette, die in seinen verschiedensten Wahlakten immer neu zu seinem Schicksal wird, sich schließlich, sobald sie die Wahl hat, aus dem Spiel verabschiedet, markiert eine Gender-typische Grenze der Erfahrung im Werk Max Frischs. Es ist die Freiheit der Frauen, die für die Männer, wenn sie ihren als Erlösungsanspruch getarnten Kontrollzwang einmal nicht ausüben können, ein völliges Rätsel bleibt. Auch hier mag man an das existenzphilosophische Grundlagenwerk der Frauenemanzipation, Simone de Beauvoirs Das andere Geschlecht, denken, doch die radikale Andersheit der Frauen in Max Frischs Euvre läßt sich mit dem philosophisch begründeten Freiheitsanspruch menschlicher Existenz für beide Geschlechter so nicht verrechnen.

Zurücktreten zudem denn auch im Lauf der 1960er Jahre die früheren Gewährsleute philosophischen Denkens : Neue Namen werden genannt oder ließen sich erschließen : vorab Ludwig Wittgenstein, dessen Konzeption des Sprachspiels ${ }^{60}$ sich geradezu wie eine Konstruktion der Poetologie des Gantenbein-Romans aus dem Jahre 1965 liest; dies gilt freilich ebenso für Schriften des Phänomenologen Willhelm Schapp, die freilich - weniger bekannt als Wittgensteins Arbeiten - doch um so prägnanter eine Formel anbieten : ,In Geschichten verstrickt'. Ob solch intertextueller Bezug zum BuchIch von Mein Name sei Gantenbein, das seine unerzählbare Erfahrung in Geschichten veräußert, als gezielte Reminiszenz zu werten wäre, läßt sich bisher nicht bestätigen ${ }^{61}$. Seit den frühen 1970er Jahren jedenfalls ergeben sich aus Frischs Teilhabe an der sogenannten ,Suhrkampkultur' weitere neue Orientierungen, vor allem durch die Schriften Walter Benjamins. Doch werden solche essayistisch gelegten Spuren ${ }^{62}$ nicht mehr in das textuelle Spiel der paradoxen Zitationen mit einbezogen. Namensnennungen dienen allenfalls der Orientierung und im Falle Walter Benjamins der Befestigung einer öffentlichen Rolle des Autors Max Frisch als Citoyen, die sich allmählich von den Verzweiflungsexperimenten und Todesgeschichten seiner Texte löst ${ }^{63}$.

24 In der autobiographischen Erzählung Montauk, 1976 erschienen, wird die Bilanz gezogen. Die Prosa übersetzt das rund ein Jahrzehnt früher entstandene Theaterstück desselben Autors Biographie. Ein Spiel, in dem Lebensentwürfe als Probehandeln auf dem (Welt-)Theater vorgeführt wurden, ins Autobiographische, mit dem Effekt freilich, daß die ,Wirklichkeit' der Verfasser-Biographie selbst zweifelhaft wird. Vielmehr durchdringen sich Imagination und Wirklichkeit auf untrennbare Weise. Und für den Autor Max Frisch konnte dies eigentlich nicht überraschend sein. Er hatte, als er seine ,autobiographische Erzählung' konzipierte, eben die Begegnung mit seinem eigenen Werk zu überstehen. In der Arbeit an der Ausgabe der Gesammelten Werke, die zudem vom Verlag als Geburtstagsgeschenk - in der persönlichen Beziehung zum Verleger wie im Marketing - deklariert war, war er seiner eigenen Vergangenheit begegnet, und die 
Frage stellte sich erneut, welcher - der gelebten oder der beschriebenen. "Ich habe mich selbst nie beschrieben. Ich habe mich nur verraten", heißt es in Montauk ${ }^{64}$. Was Montauk verrät, also dem Leser an Privatem mitteilt und damit preisgibt, sind nicht jene persönlichen Erlebnisse, für die sich literarischer Voyeurismus stets interessierte, es sind - in Inszenierungen persönlicher Konstellationen - auch Denkfiguren, deren Herkunft aus der Philosophie für den Autor freilich nur eine Potenzierung des Problems von Wirklichkeit und Imagination bedeutete. Es ist die zusätzliche Frage nach der Wirklichkeit der Wahrheit.

Eine Konstellation von drei Figuren, in der sich fünf Lebensjahrzehnte abbilden, welche gleichsam die gesamte Autorschaft Max Frischs umspannen, macht dies sinnfällig. Es ist der Besuch des Freundes W., mit Verweis auf Werner Coninx entschlüsselt, bei Max und der Frau, mit der er damals in Rom lebte, Ingeborg Bachmann. W. tritt wie eh und je als Statthalter der Philosophie auf, die Frau jedoch "hatte Philosophie studiert und über Wittgenstein geschrieben, promoviert über Heidegger" ${ }^{\text {"65 }}$. Hier wird zum einen die Beziehung zwischen dem Erzähler-Ich / Max und Ingeborg Bachmann im Dreieck von Sinnlichkeit und Philosophie (beide paradox konfiguriert in der Frauenfigur) und dem ,Todesinventar' der Schrift (konfiguriert in dem ,berühmten Schriftsteller' Max) nachgezeichnet, zum anderen wird generalisierend die Skepsis des Schriftstellers Max gegenüber W., dem Sachwalter der philosophischen ,Bildung', eingeordnet in jene umfassende Erkenntnisskepsis, wie sie sich im Werk des Autors Max Frisch sukzessive entfaltet hatte. In jener Dreier-Konstellation kommt ein wirkliches Gespräch nicht zustande. Der Repräsentant philosophischen Bildungswissens verläßt den Erzählraum, aber auch die ,Geschichte', der Lebensentwurf des Erzählers mit Ingeborg Bachmann, wird sich in eine Todesgeschichte verwandeln. Nur das Tödliche erweist sich, unter dem Horizont umfassender Skepsis, als zweifelsfreie ,Erkenntnis' ; alles andere Wissen bleibt unsicher und ist irrelevant.

Die Erzählung Der Mensch erscheint im Holozän stellt den Erkenntnisanspruch gegenüber der Welt neuerlich auf die Probe - den institutionellen, wie ihn die Wissenschaft erhebt ${ }^{66}$ und damit das Erbe der Systemphilosophie antritt, und den individuellen des Menschen, der nach dem ,wahren Leben' sucht ; in beiden Varianten aber kollabiert der Anspruch der Erkenntnis (des Mannes). Herr Geiser, die Hauptfigur von Frisches Erzählung, reduziert diesen Anspruch sogleich auf das Sammeln lexikographischer Information, also den Verzicht auf Denkordnungen, auf Modelle gar, wie sie die Philosophie entwickeln könnte. Doch auch in dieser basal reduzierten Form trügt das Wissen, und seine Bedeutungslosigkeit wird vollends offenbar, als Herrn Geiser ein Gehirnschlag ereilt. Mit der Existenz des Menschen erlischt aller Wissensanspruch gegenüber der Welt; die Archive werden bedeutungslos ${ }^{67}$. Die langjährige Auseinandersetzung des Autors Max Frisch mit dem Sündenfall der Erkenntnis mündet in die Akzeptanz dessen, was in christlichen Zeiten ,der Sünde Sold' hieß, des Todes. 


\section{NOTES}

1. Max Frisch/Friedrich Dürrenmatt: Briefwechsel. Hg. v. Peter Rüedi. Zürich: Diogenes 1998, S. 166.

2. Friedrich Dürrenmatt: Dramaturgie des Denkens. Gespräche 1988-1990. Hg. v. Heinz-Ludwig Arnold. Zürich: Diogenes 1996, S. 193.

3. Ders.: Im Bann der "Stoffe“. Gespräche 1981-1987. Hg. v. Heinz-Ludwig Arnold. Zürich: Diogenes 1996, S. 102.

4. Ders., Dramaturgie des Denkens (wie Anm. 2), S. 190.

5. Ders., Im Bann der "Stoffe“ (wie Anm. 3), S. 109. - Dagegen interessiere sich "der größte Teil der heutigen Literatur [...] nur für das, was auf der Oberfläche passiert, das Entscheidende entwickelt sich aber nicht in der hauchdünnen Schicht unseres Intellektuellseins, sondern in der ungeheuren Schicht unserer Instinkte.“ Nimmt man das mit den Äußerungen Max Frischs zusammen, so ist die Polarität in der Art konturiert, wie Frisch sie dann wiederum parodieren konnte.

6. Max Frisch: Blaubart. In: Ders.: Gesammelte Werke in zeitlicher Folge. Hg. v. Hans Mayer unter Mitw. v. Walter Schmitz. Frankfurt a. Main: Suhrkamp 1976 (Bde. I-VI)/1986 (Bd. VII) [künftig zit. als GW], Bd. VII, S. 301-403, hier S. 359, sowie Walter Schmitz: Max Frisch. Das Spätwerk (1962-1982). Eine Einführung. Tübingen: Francke 1985, S. 176.

7. Max Frisch: Homo Faber. In: GW IV, S. 5-203, hier S. 74 f.

8. Ebd., S. 22; vgl. ebd., S. 77: "Technik statt Mystik.“

9. Ders.: Stiller. In: GW III, S. 359-780, hier S. 535f.

10. Vgl. ders.: Tagebuch 1946-1949. In: GW II, S. 347-750, hier S. 629. Die dort diskutierte Forderung, die aus Goethes Wilhelm Meisters Wanderjahre durch Thomas Mann nochmals aktualisiert worden war, wird Max Frisch noch lange beschäftigen. Daß die ,Forderung des Tages' für die Literatur von Bedeutung sei, betont noch der von Frisch selbst gewählte Titel eines späten Sammelbandes seiner essayistischen Arbeiten, vgl. Walter Schmitz: Nachwort. In: Max Frisch: Forderungen des Tages. Porträts, Skizzen, Reden 1943-1982. Frankfurt a. Main: Suhrkamp 1983, S. 361-373, hier S. 361f.

11. Frauke Maria Hoß: Philosophische Elemente im Werk von Max Frisch: Grundphänomene menschlicher Existenz in den Romanen Stiller, Homo faber und Mein Name sei Gantenbein. Nordhausen: Bautz 2004, will in ihrer Studie - laut dem "Vorwort" von Thorsten Paprotny - nicht suggerieren, "daß Max Frisch ein Philosoph war"; sie beschränkt sich denn auch auf assoziative Variationen zu ,philosophiefähigen' Themen und Motiven im Werk Frischs.

12. Walter Schmitz: Max Frisch: Das Werk (1931-1961). Studien zu Tradition und Traditionsverarbeitung. Bern u.a.: Lang 1985, S. 100.

13. Max Frisch: Die Schwierigen oder J'adore ce qui me brûle. In: GW I, S. 387-599, hier S. 587. Vgl. Schmitz, Das Werk, S. 77-103 für eine umfassendere Interpretation.

14. Vielleicht überlagert von den Formulierungen, die der von Frisch damals verehrte Landsmann Albin Zollinger der eigenen Nietzsche-Rezeption gegeben hatte; vgl. Schmitz, Das Werk (wie Anm. 12), S. 101.

15. Frisch, Die Schwierigen (wie Anm. 13), S. 593.

16. Walter Schmitz, Das Werk (wie Anm. 12), S. 103. Zur ,ewigen Wiederkehr des Gleichen' vgl. auch Nietzsche-Handbuch. Leben - Werk - Wirkung. Hg. v. Henning Ottmann. Stuttgart/Weimar: Metzler 2000, S. 222-230.

17. Vgl. Walter Schmitz, Das Werk (wie Anm. 12), S. 154f. Vgl. zum Folgenden auch die Rede Vom bleichen Verbrecher in Nietzsches Also sprach Zarathustra (1883-1885).

18. Max Frisch, Tagebuch 1946-1949 (wie Anm. 10), S. 374. 
19. Hier ist eine methodische Anmerkung $\mathrm{zu}$ den - jeweils durchaus anregenden Forschungsbeiträgen notwendig, welche philosophische Kategorien unmittelbar zur Deutung von Frischs Texten nutzen. Zum einen wird damit eine ontologische Wendung vollzogen; es ist aber ein Unterschied zwischen einer im Leben vollzogenen und im literarischen Text dargestellten ,Wahl', oder ,Wiederholung'. Zwischen Literatur und Philosophie besteht stets ein Verhältnis der Intertextualität, das entsprechend $\mathrm{zu}$ interpretieren ist. Zum zweiten muß die Rekursivität gerade der Existenzphilosophie mit einbezogen werden, bereits lebensweltlich, potenziert wiederum im intertextuellen Bezug: Den existenziellen Akten wächst mit ihrer philosophischen Identifizierung eine neue Qualität zu, die wiederum - einen entsprechenden Bildungshorizont der Akteure vorausgesetzt - in die Reflexion des Handelnden eingeht; damit sind die Deutungsangebote der Philosophie immer schon historisiert.

20. Vgl. Walter Schmitz, Das Werk (wie Anm. 12), S. 191, mit den entsprechenden Hinweisen auf die Schriften Georg Simmels; generell zur vitalistischen Diskursformation vgl. Martin Lindner: Leben in der Krise. Zeitromane der Neuen Sachlichkeit und die intellektuelle Mentalität der klassischen Moderne. Stuttgart u.a.: Metzler 1994.

21. Vgl. den Abdruck in Max Frischs Andorra.Hg. v. Walter Schmitz u. Ernst Wendt. Frankfurt a. Main: Suhrkamp 1984, S. 72ff.

22. Vgl. Roland Galle: Der Existentialismus. Eine Einführung. Paderborn: Fink 2009; historisch situierend Jürgen Wertheimer: «Une saison en purgatoire ». Aspekte der Sartre-Rezeption. In: Zur literarischen Situation 1945-1949. Hg. v. Gerhard Hay. Kronberg: Athenäum 1977, S. 270-284; für das Zürcher Schauspielhaus zählte Jean-Paul Sartre in der unmittelbaren Nachkriegszeit zu den wichtigsten Autoren, vgl. Ursula Amrein: "Los von Berlin!" Die Literatur- und Theaterpolitik der Schweiz und das "Dritte Reich". Zürich: Chronos 2004, S. 529; Frisch wurde "zu den Proben von Brecht, Sartre, Lorca, Giraudoux, Claudel“ zugelassen; Frisch im Gespräch mit Horst Bienek, zit. nach: Urs Bircher: Vom langsamen Wachsen eines Zorns. Max Frisch 1911-1955. Unter Mitarb. v. Kathrin Straub. Zürich: Limmat-Verl., S. 129. - Dürrenmatt beharrt übrigens auch in der Haltung zur Existenzphilosophie auf seiner auf Welterkenntnis orientierten Position: Zu Sartre erklärt er lapidar, er könne mit dessen Philosophie "eigentlich wenig anfangen“ (Dürrenmatt, Im Bann der "Stoffe“ [wie Anm. 3], S. 227), und an anderer Stelle: "Der war ganz humorlos“ - in Dürrenmatts Weltbild kein Kompliment (ebd., S. 101).

23. Walter Schmitz: Neun Thesen zu Andorra. In: Max Frischs Andorra (wie Anm. 21), S. 143-159, hier S. 147. - Die Sartre-Aufführungen am Züricher Schauspielhaus - am 12. Oktober 1944 die deutschsprachige Erstaufführung Die Fliegen, Mitte März 1951 in einem Gastspiel der Basler Komödie nicht nur Die ehrbare Dirne/ La Putain Respectueuse, sondern auch Geschlossene Türen/Huis Clos - hat er nicht nur verfolgt, sondern für seine Kritik an der Züricher Theateröffentlichkeit beruft er sich auch auf Analogien im Umgang mit Sartre, der mal skandalisiert, dann bejubelt werde (vgl. Kleines Memorandum zu Graf Öderland. In: Max Frisch: Jetzt ist Sehenszeit. Briefe, Notate, Dokumente 1943-1963. Frankfurt a. Main: Suhrkamp 1998, S. 95-101, hier S. 96). Doch auch Fehlings Berliner Inszenierung von Sartres Die Fliegen, die im Herbst 1947 intensiv diskutiert wurde, wird vermerkt (vgl. ebd., S. 224). - Und gelegentlich findet sich auch die Gesprächsnotiz: "Camus und Dürrenmatt" (Tagebuch Griechenland, Frühling 1957. In: Ebd., S. 158-170, hier S. 166), freilich gefolgt von dem Lektürenotat: "Camus LA CHUTE zu Ende gelesen, unbefriedigt" (Eintrag vom 15. Mai, ebd., S. 168), immerhin lohnend genug um "[...] Notizen darüber [...]" (ebd.) festzuhalten.

24. Vgl. Jean-Paul Sartre: Betrachtungen zur Judenfrage. In: Ders.: Drei Essays. Mit e. Nachw. von Walter Schmiele. Frankfurt a. Main: Ullstein 1960, S. 108-190. Sartre hält bereits am Beginn seiner Argumentation fest: "Nicht die Erfahrung schafft den Begriff des Juden, sondern das Vorurteil fälscht die Erfahrung. Wenn es keinen Juden gäbe, der Antisemit würde ihn erfinden.“ (S. 111) Im einschlägigen Kapitel von Sartres Hauptwerk Das Sein und das Nichts wird die umfassende Konzeption des Hasses, wie ihn der Antisemit exemplarisch vertritt, dargestellt: Der Hassende 
"will eine Welt verwirklichen, in der es den Anderen nicht gibt“; zit. nach der konzisen Skizze bei Schmiele: Nachwort. In: Ebd. S. 191-206, hier S. 199.

25. Vgl. noch immer Karl Löwith: Von Hegel zu Nietzsche. Der revolutionäre Bruch im Denken des neunzehnten Jahrhunderts. Frankfurt a. Main: Fischer 1969.

26. Schmitz, Spätwerk (wie Anm. 6), S. 42. Vgl. auch Régine Battiston-Zuliani: Lectures de l'identité narrative.Max Frisch, Ingeborg Bachmann, Marlen Haushofer, W.G. Sebald. [Paris]: Orizons 2009, S. 48.

27. Der Terminus ,essentially contested concept' wurde eingeführt von Walter B. Gallie: Essentially Contested Concepts. In: Proceedings of the Aristotelian Society. Bd. 56 (1956), S. 167-198.

28. Vgl. Galle, Existentialismus (wie Anm. 22), S. 23ff. zu den letztgenannten Schlüsselkonzepten Sartres. - Weiteres: "Die Würde des Menschen [...] besteht in der Wahl", hatte bereits das Tagebuch 1946-1949 (wie Anm. 10, S. 488) formuliert, auch hier wiederum in eigenständiger Übernahme existenzphilosophischer Diktion, vgl. zu Kierkegaard: Lexikon Existenzialismus und Existenzphilosophie. Hg. v. Urs Thurnherr u. Anton Hügli. Darmstadt: WBG 2007, S. 282f. - Die Konzeption des ,Anderen', die mit der ,Geschichte vom andorranischen Juden' ebenfalls im Tagebuch 1946-1949 anhebt und mit ,neuromantischen' - so im Text Die andere Welt (1945) - und Jungianischen Konzepten wie in Bin oder Die Reise nach Peking bricht, wird bis zur Namenschiffre ,Andri' in Andorra fortgeführt; sie dürfte tatsächlich bei Sartre angeschlossen haben; vgl. Lexikon Existentialismus, S. 22-25, sowie auch zur ,Scham' die Hinweise bei Galle, Existentialismus (wie Anm. 22), S. 37.

29. Max Frisch, Stiller (wie Anm. 9), S. 421. Vgl. im Folgenden auch Battiston, Lectures (wie Anm. 26), S. $49 \mathrm{ff}$.

30. Vgl. Norbert Heinel: Der Begriff der Wiederholung bei Sören Kierkegaard. Diss., Wien 1975 sowie Dorothea Glöckner: Kierkegaards Begriff der Wiederholung. Eine Studie zu seinem Freiheitsverständnis. Berlin/New York: de Gruyter 1998.

31. Lexikon Existentialismus (wie Anm. 28), S. 296. Vgl. auch Doris Kiernan: Existentiale Themen bei Max Frisch. Die Existentialphilosophie Martin Heideggers in den Romanen Stiller, Homo faber und Mein Name sei Gantenbein. Berlin/New York: de Gruyter 1978, S. 158ff. u.ö.

32. Jean-Paul Sartre: Ist der Existentialismus ein Humanismus? In: Sartre, Essays (wie Anm. 24), S. 7-51, hier S. 17; vgl. Materialien zu Max Frisch Stiller. 2 Bde. Hg. v. Walter Schmitz. Frankfurt a. Main: Suhrkamp 1978, Bd. 1, S. 12. - Peter von Matt: Die tintenblauen Eidgenossen. Über die literarische und politische Schweiz. München/Wien: Hanser 2001, bes. S. 232, verweist hier ebenfalls auf die "Dezisionskultur des französischen Existenzialismus der vierziger Jahre“: "Der unvermittelte Aufbruch, der Wille zu einem Dasein ohne Kompromiß, die rücksichtslose Selbstsetzung [...], dies alles waren epochale Chiffren der Camus- und Sartre-Zeit. Max Frisch verwirklichte sie in seinem Werk gleichzeitig mit den Franzosen und auf seine eigene Art.“ Schmiele macht darauf aufmerksam, daß der Selbstentwurf des Menschen bei Sartre eben nicht mit einer ,Entschlossenheit' verwechselt werden darf, wie sie in Nietzsches Denken angelegt und in einer plakativ aktualisierenden Rezeption Heideggers gerechtfertigt wurde; vgl. Schmiele, Nachwort (wie Anm. 24), S. 194ff. - Vor allem wäre hier noch auf die zentrale ,motivlose Tat' im Bühnenstück Graf Öderland zu verweisen; vgl. Iris Roebling: Der Staatsanwalt mit der Axt: Graf Öderland. In: Dies.: ,Acte Gratuit'. Variationen einer Denkfigur von André Gide. München: Fink 2009, S. 215-218.

33. Max Frisch, Stiller (wie Anm. 9), S. 499 f.

34. Ebd., S. 466; vgl. ebd., S. 467. - Zur Reproduktion von Thomas Manns Zauberberg im sehr viel bescheideneren Milieu von Julikas Kur am selben Ort vgl. Schmitz, Das Werk (wie Anm. 12), S. 268 und Max Frisch: Spuren meiner Nicht-Lektüre. In: Walter Schmitz: Materialien zu Max Frisch Stiller. Bd. I. Frankfurt a. Main: Suhrkamp 1978, S. 341f.

35. Vgl. Max Frisch, Stiller (wie Anm. 9), S. 749.

36. Vgl. Glöckner, Kierkegaards Begriff der Wiederholung (wie Anm. 30), S. 6 u.ö., insbesondere auch $\mathrm{zu}$ den Verbindungslinien zwischen Wiederholung, Freiheit, Liebe und Autorschaft in 
Kierkegaards Denken. Zur von mir hier nur skizzierten Deutung des Romans ausführlicher Schmitz, Das Werk (wie Anm. 12), S. 251-255, mit dem Fazit, Frischs Roman Stiller handele "von den Schwierigkeiten, sich seiner ,wahren' Existenz zu versichern im Zeitalter der programmatischen Existenzphilosophie“ (S. 255). Dementsprechend bilden die Motti aus Kierkegaards Entweder-Oder, die Stillers "Aufzeichnungen aus dem Gefängnis“ vorangestellt sind, nicht den Schlüssel, sondern den Kontrapunkt zu diesen Aufzeichnungen. Nicht weiterführend: Hoß, Philosophische Elemente (wie Anm. 11), S. 53-65.

37. Die Figuren umkreisen diese Einsicht in ihren Deutungen der Situation, Rolf mit Anleihen bei Kierkegaard (Frisch, Stiller [wie Anm. 9], S. 750ff.), Stiller lakonisch mit der Formel: "Wie verhält man sich unter einem Fluch?" (Ebd. S. 753).

38. Die Struktur des Romans Stiller insgesamt geht von dem Spiel mit Autorschaften aus, das Kierkegaard in seinen Schriften immer wieder inszeniert, und hier insbesondere in EntwederOder;in Kierkegaards Buch folgt auf die Aufzeichnung des anonymen Schreibers A der zweite Teil, für den der vernünftige Gerichtsrat Wilhelm zeichnet - eine Struktur, die zurück verweist auf die Entwicklung in Goethes CEuvre von der Selbstmorderzählung der Leiden des jungen Werthers, also einem exemplarischen Text der Verzweiflung, zu dem Bildungsangebot von Wilhelm Meisters Lehrjahren. Für den Roman Stiller wird freilich deutlich, daß es sich auch hier um eine Form von kultureller Übersetzung als Reproduktion handelt. Rolf ist eben kein Philosoph, sondern nur ein Leser philosophischer Werke, ohne daß auch nur festzustellen wäre, welcher. Frischs Kritik an der Reprise des Bildungsbürgertums in den 1950er Jahren, wie sie ihm eben gerade im Schweizer, und hier insbesondere im Zürcher kulturellem Milieu entgegentrat, muß hier mit in Betracht gezogen werden. Sie kumuliert dann in seiner Abrechnung mit dem Germanisten Emil Staiger, einem ihm persönlich gut bekannten Repräsentanten dieses Milieus. Werkgeschichtlich noch bedeutsamer als der Zürcher Literaturstreit ist die Revision des Verhältnisses zu W. in Montauk;dazu vgl. unten die entsprechenden Passagen.

39. Max Frisch, Stiller (wie Anm. 9), S. 733.

40. Ebd., S. 734.

41. Vgl. ebd., S. $750 f$.

42. Vgl. ebd., S. 751.

43. Ebd., S. 764.

44. Vgl. ebd., S. 764.

45. Vgl. Angela Maria Kochs: Chaos und Individuum. Robert Musils philosophischer Roman als Vision der Moderne.Freiburg i.Br.: Alber 1996.

46. Max Frisch, Homo Faber (wie Anm. 7), S. 70. Vgl. ebd., S. 89 das Tanz-Motiv: "[...] ihre heutigen Tänze, [...] diese existentialistische Hopserei, wo jeder für sich allein tanzt“. - Volker Schlöndorff hat in seiner von Frisch immerhin autorisierten Verfilmung des Romans generell die parodistischen Züge zugunsten einer plakativen Existenzparabel verfehlter - männlicher Existenz zurückdrängt. Schon bei einem ihrer ersten Auftritte liest Sabeth in einem Exemplar von Camus' Der Fremde.

47. Vgl. nochmals Wertheimer, Sartre-Rezeption (wie Anm. 22), sowie zu den Textstrategien, Galle, Existentialismus (wie Anm. 22), S. 124 u.ö.

48. Bircher, Vom langsamen Wachsen (wie Anm. 22), S. 126.

49. Vgl. Max Frisch: Don Juan oder Die Liebe zur Geometrie. In: GW III, S. 95-167, hier S. 131. Vgl. Knut Radbruch: Mathematische Spuren in der Literatur. Darmstadt: WBG 1997, S. $213 \mathrm{ff}$.

50. Vgl. Walter Rehm: Kierkegaard und der Verführer. München: Rinn 1949, bes. S. 75ff., sowie S. 229ff. zur ",Wiederholung' des Verführers". Vgl. auch meine Skizze: "Don Juans Dasein in der europäischen Literatur: Kapitel aus der Geschichte einer Symbolgestalt.“ In: Frischs Don Juan oder Die Liebe zur Geometrie. Frankfurt a. Main: Suhrkamp 1985, S. 208-262, insb. S. $220 \mathrm{ff}$.

51. Max Frisch, Tagebuch 1946-1949 (wie Anm. 10), S. 379. 
52. Vgl. die hier anzitierte Formulierung im Titel des - Frisch wahrscheinlich bekannten Hauptwerks von Ludwig Klages: Der Geist als Widersacher der Seele. Leipzig: Barth 1929.

53. Vgl. die Begegnung mit dem ,Geist' in Frischs Skizze über den Rechtsanwalt Schinz. In: Tagebuch 1946-1949 (wie Anm. 10), S. 723-749, dazu Schmitz, Das Werk (wie Anm. 12), S. 19f. Fabers Fremdheit in der Welt wäre mit einem Rückverweis auf Camus' L'Étranger ebensowenig erklärt wie Stillers Existenzdilemma.

54. Max Frisch, Stiller (wie Anm. 9), S. 458.

55. Vgl. ebd., S. 777ff.

56. Vgl. dazu auch Klaus Schuhmacher: "Weil es geschehen ist“. Untersuchungen zu Max Frischs Poetik der Geschichte. Königstein/Ts.: Hain 1979, S. 139ff.

57. Max Frisch: Mein Name sei Gantenbein. In: GW V, S. 5-320, hier S. 64.

58. Max Frisch, Tagebuch 1946-1949 (wie Anm. 10), S. 488.

59. Vgl. dazu Schmitz, Spätwerk (wie Anm. 6), S. 7.

60. Vgl. Kurt Wuchterl: Struktur und Sprachspiel bei Wittgenstein. Frankfurt a. Main: Suhrkamp 1969; Reinier Franciscus Beerling: Sprachspiele und Weltbilder: Reflexionen zu Wittgenstein. Freiburg i. Br./München: Alber 1980. Vgl. außerdem Hans-Johann Glock: Wittgenstein-Lexikon. Darmstadt: WBG 2000, S. 325-330.

61. Vgl. Schmitz, Spätwerk (wie Anm. 6), S. 40ff., vgl. dazu die Bände von Wilhelm Schapp In Geschichten verstrickt. Zum Sein von Mensch und Ding (1953) und Philosophie der Geschichten (1959).

62. Vgl. sein Bekenntnis zu einer linken Utopie, wie er sie etwa in seiner Friedenspreisrede formuliert hat: Max Frisch: Wir hoffen. Rede zur Verleihung des Friedenspreises des deutschen Buchhandels 1976. In: Frisch, Forderungen des Tages (wie Anm. 10). S. 332-342, insb. S. 336, den Abschnitt Wozu die Utopie?.

63. Vgl. dazu meine Studie: Max Frisch: Schwarzes Quadrat - Eine Poetik im Zitat. In: Max Frisch. Citoyen und Poet. Hg. v. Daniel De Vin. Göttingen: Wallstein 2011, S. 56-71, S. 68, zu einem offenkundig von Max Frisch lancierten Benjamin-,Zitat'.

64. Max Frisch: Montauk. In: GW VI, S. 617-754, hier S. 720.

65. Ebd., S. 648 (Hervorhebung W.S.).

66. Vgl. dazu die anregenden Perspektiven bei Georg Braungart: "Katastrophen kennt allein der Mensch, sofern er sie überlebt": Max Frisch, Peter Handke und die Geologie. In: Figurationen der literarischen Moderne. Festschrift für Helmuth Kiesel. Hg. v. Carsten Dutt u. Roman Luckscheiter. Heidelberg: Winter 2007, S. 23-41.

67. Vgl. Barbara Schmenk: "Entropie der Archive.Todesarten in Max Frischs Der Mensch erscheint im Holozän“. In: Die Medialität des Traumas. Eine Archäologie der Gegenwartskultur. Hg. v. Ralph Köhnen u.a. Frankfurt a. Main u.a.: Lang 2006, S. 175-191, umfassender zur thematischen Entwicklung im Werk Frischs seit 1945 Richard Millington: Configurations of Memory Crisis in the Narrative Prose of Max Frisch. In: Erinnerungskrisen - Memory Crises. Hg. v. Franz-Josef Deiters. Freiburg i. Br.: Limbus 2008, S. 181-198.

\section{RÉSUMÉS}

Max Frisch a non seulement nié avoir une prétention philosophique, mais il a aussi délégué ce refus à ses personnages, en particulier à l'ingénieur Walter Faber. Toutefois ce refus est contredit d'une part par de nombreuses sources qui relient les textes de Max Frisch à des œuvres 
philosophiques, d'autre part, par un intérêt constant pour des questions débattues dans la philosophie contemporaine; enfin, le fameux scepticisme de Max Frisch se révèle être la face cachée d'une fascination pour la connaissance. La contribution propose par conséquent un développement en trois étapes. La première partie éclaire les différentes facettes de la critique philosophique chez Max Frisch; dans une deuxième partie, sont identifiés les liens avec la philosophie sur la base des résultats des recherches actuelles. La troisième partie représente le point culminant. D'une part, dans un axe qui va de Don Juan ou l'amour de la géométrie à travers Stiller et Homo Faber jusqu'à Montauk, est retracée la triade sensualité, philosophie et « inventaire de la mort » de l'écriture. D'autre part le scepticisme de l'écrivain Max Frisch envers la philosophie est intégré de manière générale dans le dispositif de scepticisme de la connaissance, qui est développé dans l'œuvre de cet auteur.

Nicht nur Max Frisch selbst hat sich dem philosophischen Erkenntnisanspruch verweigert, sondern er hat diese Weigerung auch an seine Figuren, insbesondere an den Ingenieur Walter Faber, delegiert. Allerdings steht dieser Verweigerung eine Vielzahl von Quellenbeziehungen, die Max Frischs Texte mit philosophischen Werken verbinden, entgegen, des weiteren ein ständiges Interesse an Themen, die in der zeitgenössischen Philosophie verhandelt werden; und schließlich läßt sich auch die notorische Erkenntnisskepsis Max Frischs als die Kehrseite einer Faszination an sicherer Erkenntnis entziffern. Der Beitrag geht demnach in drei Schritten vor. In einem ersten Abschnitt werden die verschiedenen Facetten der Philosophiekritik bei Max Frisch beleuchtet, in einem zweiten werden auf der Basis bisheriger Forschungsergebnisse die Verbindungslinien zur Philosophie aufgezeigt. Das Schwergewicht aber liegt auf dem dritten Abschnitt. Hier wird zum einen in einem Bogen von Don Juan oder die Liebe zur Geometrie über Stiller und Homo Faber bis zu Montauk das Dreieck von Sinnlichkeit, Philosophie und dem 'Todesinventar' der Schrift nachgezeichnet, zum anderen wird in einem generalisierenden Schritt die Skepsis des Schriftstellers Max Frischs gegenüber der Philosophie in ein Dispositiv der Erkenntnisskepsis, wie es im Werk dieses Autors sukzessive entfaltet wird, eingeordnet.

Not only Max Frisch himself denied the philosophical claim for knowledge, he also delegated this refusal to his personae, in particular to the engineer Walter Faber. However, contrary to this refusal stand the various relations to the sources that link Max Frisch's texts with philosophical works. In addition, his writing shows an ongoing interest in topics of contemporary philosophy. Finally, the notorious scepticism towards knowledge can be deciphered as the downside of such a fascination with knowledge. The article will analyze this in three steps. In a first part, the different facets of Frisch's philosophical criticism will be analyzed. Secondly, the relations to philosophy will be shown on the basis of previous researches. The major focus, though, is on the third part. On the one hand, the triangle of sensuality, philosophy, and the 'deathly' inventory of scripture will be traced using Don Juan oder die Liebe zur Geometrie, Stiller,Homo Faber and Montauk. On the other hand, in a generalizing step, the writer Max Frisch's scepticism concerning philosophy will be put in the context of a dispositif of scepticism towards knowledge alltogether.

\section{INDEX}

Mots-clés : éthique politique, Heimat, suissitude 
AUTEURS

WALTER SCHMITZ

Europa Zentrum, Universität Dresden 